\title{
INTERFACE SHARPENING IN TWO-PHASE FLOWS BASED ON PRIMITIVE SUB-CELL RECONSTRUCTIONS
}

\author{
IGOR MENSHOV ${ }^{1}$, CHAO ZHANG² AND PAVEL ZAKHAROV ${ }^{3}$ \\ ${ }^{1}$ Keldysh Institute of Applied Mathematics, RAS \\ Miusskaya sq. 4, Moscow, Russia \\ menshov@kiam.ru https://istina.msu.ru/profile/imenshov/ \\ ${ }^{2}$ Institute of Applied Physics and Computational Mathematics, Beijing 100094, China \\ zhang-c@mail.ru \\ ${ }^{3}$ VNIIA ROSATOM \\ Sushchevskaya 22, Moscow, Russia \\ jaquecostou@mail.ru
}

Key words: two-phase flow, diffuse interface, sub-cell reconstruction, composite Riemann problem.

\begin{abstract}
The paper addresses a novel interface-capturing approach for two-phase flows governed by the five-equation diffuse interface model. To suppress the numerical diffusion of the interface, we introduce a primitive sub-cell reconstruction based on volume fractions in neighbouring cells. This reconstruction gives rise to a Riemann problem (CRP) with an additional contact discontinuity, so-called composite Riemann problem, which is stated on mixed cell faces. The CRP solution is used to calculate the numerical flux across cell faces of mixed cells with taking into account the interface reconstructed patterns. A hybrid HLLHLLC method is incorporated to approximate the solution of the CRP. The proposed approach is shown to effectively reduce the interface numerical diffusion without introducing spurious oscillations. Its performance and robustness is examined by $1 \mathrm{D}$ and $2 \mathrm{D}$ numerical tests.
\end{abstract}

\section{INTRODUCTION}

In this paper we consider a numerical method for compressible two-phase flow simulations. It is an Eulerian interface capturing method where the material interface is allowed to diffuse in space forming a narrow mixture zone. The diffuse zone is a typically numerical effect that should be minimized as much as possible in calculations. With this aim, many interface sharpening techniques have been developed in literature, among which are artificial interface compression [1], anti-diffusion correction algorithm [2], THINC scheme [3].

Apart from these techniques there is another method which can be considered as a hybrid interface tracking-capturing method. Proposed in [4] for the equilibrium P-T model, this method involves two steps - sub-cell reconstruction and flux approximation which accounts 
for the reconstructed interface in mixed cells. If the interface crosses the cell face, the flux is approximated on the solution of composite Riemann problem (CRP) [4] which, besides the face-related initial discontinuity in one pure fluid, involves also a point of contact with another fluid located at some distance from the face. In [5], the CRP solution was implemented in the Godunov method for the five-equations two-phase flow model of Allaire et al. [6]. A 1D discontinuous step-wise function is used here to approximate the volume fraction distribution in mixed cells in each coordinate direction. The CRP serves to take into account the effect of in-cell discontinuity on the numerical flux.

The purpose of the present paper is two-fold: to extend the approach of [4] to the fiveequation model and formalize the algorithm of sub-cell reconstruction. In the original work [4], the cell connectivity is separated into two types: "mixed-mixed" and "mixed-pure". For each type, the sub-cell reconstruction is performed differently, with being no clear choice of the reconstruction pattern in the "mixed-mixed" case. We propose a simple rule for the pattern reconstruction based on transparent conditions. This rule works for the both types and uniquely defines the reconstruction pattern by using only values of cell volume fractions. Moreover, an effective HLL-HLLC method is proposed to approximate the solution of the CRP.

\section{MATHEMATICAL MODEL AND BASELINE NUMERICAL METHOD}
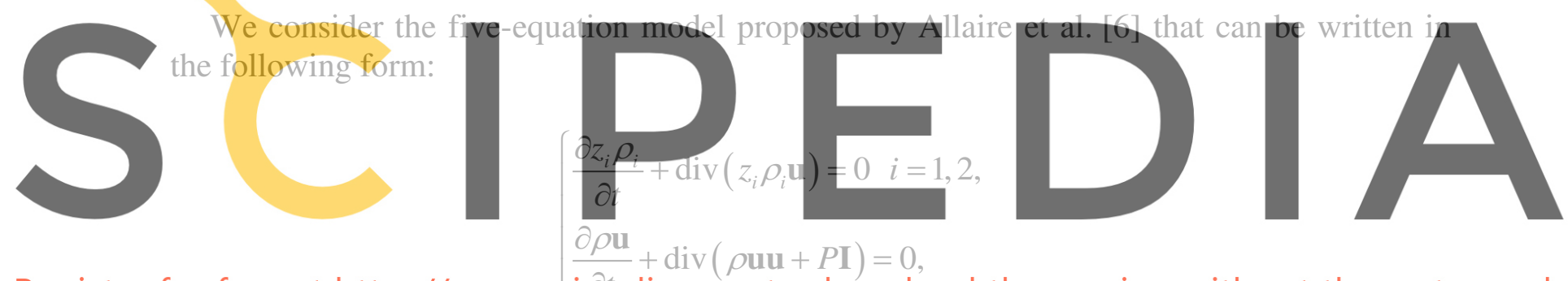

Register for free at https//www.scipedia.com to download the version without the watermark

$$
\left\{\begin{array}{l}
\frac{\partial \rho e}{\partial t}+\operatorname{div}(\rho H \mathbf{u})=0 \\
\frac{\partial z}{\partial t}+\operatorname{div}(\mathbf{u} z)-z \operatorname{div}(\mathbf{u})=0
\end{array}\right.
$$

where $z$ denotes the volume fraction, $\rho$ is the density, $\mathbf{u}$ is the velocity vector, $e$ is the specific internal energy, $P$ is the pressure, $H$ is the specific total enthalpy. In the last equation of the system (1) we use $z$ instead of $z_{1}$ for the sake of simplicity. This equation is in fact a modification of the advection equation for the volume fraction of the phase $i=1$. Such a form naturally maintains the well-balancing property when the divergence term is discretized as the summation of fluxes estimated with the proposed CRP interface-sharpening approach.

The model (1) is the reduced Baer-Nunziato model with single velocity and single pressure for mixture derived by A.K. Kapila et al. [7]. It can be used with the equation of state in the form of generalized Mie-Gruneisen equation, 


$$
P_{i}\left(\rho_{i}, \rho_{i} \varepsilon_{i}\right)=\left(\gamma_{i}\left(\rho_{i}\right)-1\right) \rho_{i} \varepsilon_{i}-\gamma_{i}\left(\rho_{i}\right) \pi_{i}\left(\rho_{i}\right)
$$

where $\gamma_{i}$ and $\pi_{i}$ are dependent on the density of the corresponding material. Allaire et al. [6] showed that constant pressure equilibrium is properly preserved if the isobaric closure is employed, $P_{1}=P_{2}=P$, in the cells containing two fluids.

Defining $\delta_{i}=\left(\frac{\partial \rho_{i} \varepsilon_{i}}{\partial \rho_{i}}\right)_{P_{i}}$ and $\xi_{i}=\left(\frac{\partial \rho_{i} \varepsilon_{i}}{\partial P_{i}}\right)_{\rho_{i}}$, the equation of state for the mixture takes the following form:

$$
P\left(\rho_{1}, \rho_{2}, \rho \varepsilon, z\right)=\frac{\rho \varepsilon}{\xi}-\frac{1}{\xi} \sum_{i=1}^{2} z_{i} \gamma_{i}\left(\rho_{i}\right) \pi_{i}\left(\rho_{i}\right) \xi_{i}\left(\rho_{i}\right)
$$

with $\xi\left(\rho_{1}, \rho_{2}, z\right)=\sum_{i=1}^{2} z_{i} \xi_{i}\left(\rho_{i}\right)$ and $\xi_{i}\left(\rho_{i}\right)=\frac{1}{\gamma_{i}\left(\rho_{i}\right)-1}$.

\section{The sound speed of each pure material is calculated by}

$$
c_{i}=\sqrt{\left(h_{i}-\delta_{i}\right) / \xi_{i}}
$$

\section{with $\delta_{i}\left(\rho_{i}\right)=-\frac{\gamma_{i}^{\prime}\left(\rho_{i}\right)}{\left[\gamma_{i}\left(\rho_{i}\right)-1\right]^{2}}\left[P_{i}+\pi_{i}\left(\rho_{i}\right)\right]+\frac{\gamma_{i}\left(\rho_{i}\right) \pi_{i}^{\prime}\left(\rho_{i}\right)}{\gamma_{i}\left(\rho_{i}\right)-1}$, and $h_{i}$
entalphy.
The sound speed of the nixixure is calculated by
$c=\sqrt{\sum^{2} y_{i} \xi_{i}\left(\rho_{i}\right) c_{i}^{2} / \xi\left(\rho_{1}, \rho_{2}, z\right)}$
for free at https//www.scipedia.Com to download the vers}

The system of equations (1) can be recast in the following vector form:

$$
\frac{\partial \mathbf{q}}{\partial t}+\frac{\partial \mathbf{f}_{i}(\mathbf{q})}{\partial x_{i}}=\mathbf{s}(\mathbf{q})
$$

where $\mathbf{q}$ is the conservative vector, $\mathbf{q}=\left[\rho_{1} z_{1} \rho_{2} z_{2} \rho u \rho v \rho w \rho e z\right]^{\mathrm{T}}, \mathbf{s}(\mathbf{q})=\left[\begin{array}{lllllll}0 & 0 & 0 & 0 & 0 & 0 & z \operatorname{div}(\mathbf{u})\end{array}\right]$.

The present numerical method for multi-material fluid flows is based on the Godunov finite volume discretization of the single-material Euler equations. We use a stationary computational grid consisting of non-overlapping polyhedrons (cells) that cover the computational domain. The spatial discretization of Eq. (6) without the source term $\mathbf{s}(\mathbf{q})$ is performed with the finite volume method, which yields with the explicit time marching scheme the following discrete equations:

$$
\mathbf{q}_{i}^{n+1}=\mathbf{q}_{i}^{n+1}-\frac{\Delta t}{V_{i}} \sum_{\sigma}\left(\mathbf{f}_{k} n_{k}\right)_{\sigma} S_{\sigma}
$$


where the subscripts $i$ and $\sigma$ denote the cell and its face, respectively, $V_{\mathrm{i}}$ is the volume of the $i$-th cell, $S_{\sigma}$ is the face area, and $n_{k}, k=1,2,3$ are the components of the outward unit normal to the face. Each term in the summation at the right-hand side of Eq. (7) can be expressed in terms of one flux vector $\mathbf{F}$ as follows:

$$
\mathbf{f}_{k} n_{k}=\mathbf{T}^{-1} \mathbf{F},
$$

where $\mathbf{T}$ is the transforming matrix from the absolute system of coordinates to a coordinate system associated with the face. The vector $\mathbf{F}=\mathbf{F}(\mathbf{Q}), \mathbf{Q}=\mathbf{T} \mathbf{q}$, has the form of a locally 1D flux in the direction of the face outward normal,

$$
\mathbf{F}=\mathbf{F}(\mathbf{Q})=\left(\rho_{1} z_{1} v_{n}, \rho_{1} z_{1} v_{n}, \rho v_{n}^{2}+p, \rho v_{n} v_{k}, \rho v_{n} v_{l}, \rho v_{n} E+p v_{n}, v_{n} z\right)^{T}
$$

where the subscripts $n, k$, and $l$ indicate normal and tangential components of the velocity vector. Thus, the system of discrete equations takes the following form:

$$
\mathbf{q}_{i}^{n+1}=\mathbf{q}_{i}^{n}-\frac{\Delta t}{V_{i}} \sum_{\sigma} T_{\sigma}^{-1} \mathbf{F}_{\sigma} S_{\sigma} .
$$

The numerical flux $\mathbf{F}_{\sigma}$ depends on the state of the medium in the cells adjacent to the face $\sigma$

$$
\mathbf{F}_{\sigma}=\mathbf{F}_{\sigma}\left(\mathbf{z}_{i}^{\sigma}, \mathbf{z}_{\sigma(i)}^{\sigma}\right)
$$

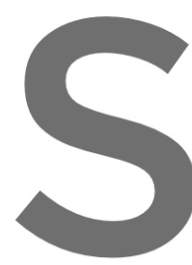

\section{where $\sigma(i)$ denotes a cell means that the primitive \\ face. We use a predicto - enhance the space-tine
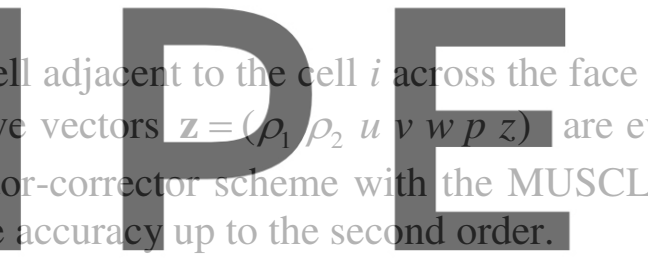
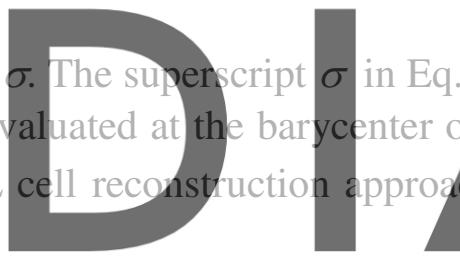

To approximate

numeric

flux, the

the face-basec

Riemann problem solution is

Register foppdreentedittps//www.scipedia.com to download the version without the watermark

$$
\mathbb{F}_{\sigma}=\mathbb{F}\left[\mathbb{Z}^{R}\left(\mathbb{Z}_{i}^{\sigma}, \mathbb{Z}_{\sigma(i)}^{\sigma}\right)\right]
$$

where the superscript $\mathrm{R}$ indicates the Riemann problem solution, and $\mathbf{Z}=T \mathbf{z}$. This solution is approximately defined with the HLLC method. With proper estimation of the shockwaves and contact wave, HLLC solver resolves discontinuities sharply, and isolated shockwaves and contacts exactly. The shock wave velocities $s_{L}$ (leftmost) and $s_{R}$ (rightmost) are estimated by the method of Einfeldt et al. [8]:

$$
s_{L}=\min \left(\bar{u}-\bar{c}, u_{L}-c_{L}\right), s_{R}=\min \left(\bar{u}+\bar{c}, u_{L}+c_{L}\right),
$$

where $\bar{u}$ and $\bar{c}$ are average parameters of the states on both sides and can be estimated by an arithmetic or Roe average, we adopt the latter in this paper.

The speed of the intermediate contact wave is estimated by the method of Batten et al. [9]:

$$
s_{*}=\frac{P_{R}-P_{L}+\rho_{L} u_{L}\left(s_{L}-u_{L}\right)-\rho_{R} u_{R}\left(s_{R}-u_{R}\right)}{\rho_{L}\left(s_{L}-u_{L}\right)-\rho_{R}\left(s_{R}-u_{R}\right)}
$$


The above flux approximation can be applied straightforwardly to solve multi-material fluid flow modeled by Eq. (1). Thus, calculations in the whole domain can be executed in the same manner, both in pure and mixed cells. We refer this straightforward extension of the conventional flux approximation to the multi-material case as a standard flux approximation.

The standard flux approximation results in significant numerical smearing of material interfaces as it does not take into account the presence of the sharp material interface inside mix cells. Thus, if a non-zero mass flux appears from a mix cell to an adjacent pure cell, it inevitably results in non-zero mass fluxes of all components containing in the mixed cell.

As a solution to this problem, we suggest to modify the flux approximation for those cell faces that border at least one mixed cell. This is realized on the base of a simple pattern of the sub-cell interface reconstruction with subsequent flux approximation on the solution to the CRP arising on the face due to this reconstruction. We call this method as CRP-based flux approximation.

\section{SUB-CEIL RECONSTRUCTION AND CRP-BASED FLUX APPROXIMATION}

The CRP flux approximation is applied to only the mixed cells or the cells bordering a mixed cell. Mathematically, the mixed cell is identified by the condition $\eta<z<1-\eta$, where $\eta$ is a small positive number. Consider a cell and its neighbor cell with the volume fractions $z$ and $z_{n}$, respectively (Fig. 1). The subscript $n$ indicates the neighbour cell.

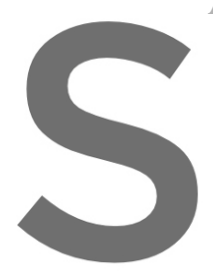
Assume that at least one of these cells is mixed.
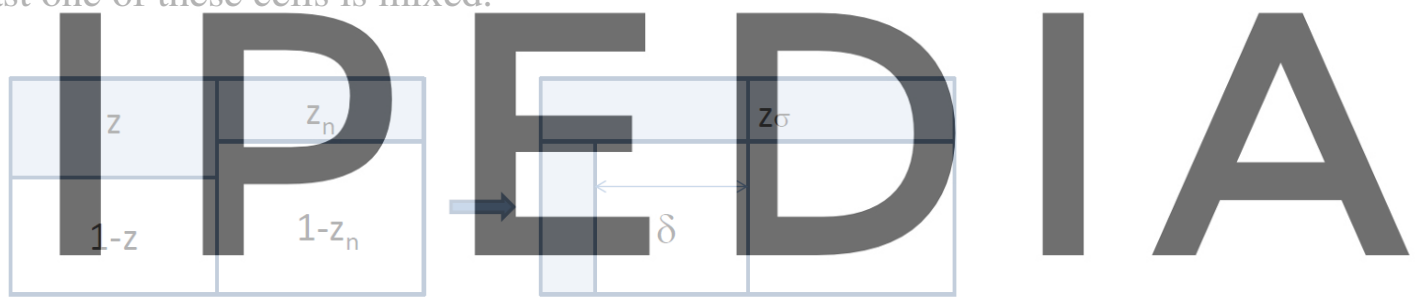

Register for free at https//www.scipedia.com to download the version without the watermark

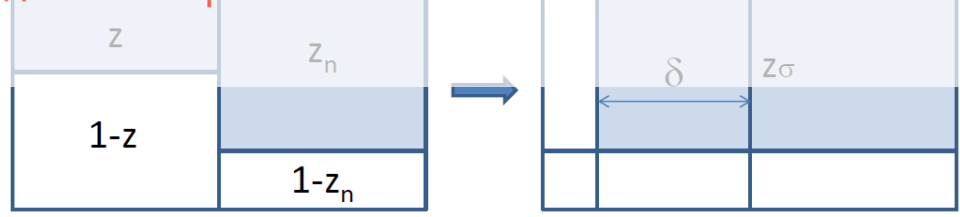

Fig. 1. Patterns of the sub-cell interface reconstruction: $z+z_{n} \leq 1$ (top), $z+z_{n} \geq 1$ (bottom).

The interface crosses the face between the cells so that some part of the face is in the first component and another is located in the second component. By analogy with volume fraction, we introduce surface fraction $z_{\sigma}$ to charterize the phase distribution on the face, and assume that this parameter is a function of the volume fractions in neigbouring cells, $z_{\sigma}=\phi\left(z, z_{n}\right)$.

The function $\phi(x, y)$ is defined in $0 \leq x \leq 1,0 \leq y \leq 1$ and should satisfy the following properties: 


$$
\begin{aligned}
& \phi(0, y)=\phi(x, 0)=0, \\
& \phi(1, y)=\phi(x, 1)=1, \\
& \phi(x, x)=x, \\
& \phi(x, y)+\phi(1-x, 1-y)=1 .
\end{aligned}
$$

The first two conditions mean that the interface does not cross the cell face when one of the components vanishes in one of the cells. The third and forth describe the symmetric property.

Simple choice for the function $\phi(x, y)$ is as follows :

$$
\phi(x, y)=\left\{\begin{array}{l}
\min (x, y), \text { if } x+y<1 \\
\max (x, y), \text { if } x+y>1 \\
0.5(x+y), \text { if } x+y=1
\end{array}\right.
$$

This function is discontinuous at points $x+y=1$. Another choice is a continuous function defined as ${ }^{1}$
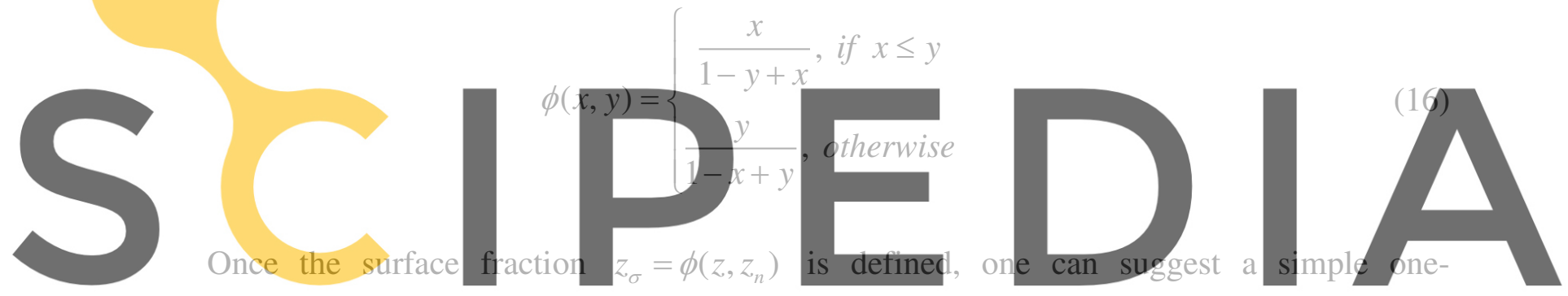

dimensional pattern of the sub-cell interface reconstruction as shown in Fig. 1. It is

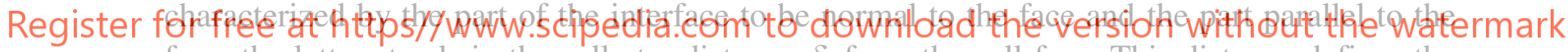
face; the latter stands in the cell at a distance $\delta$ from the cell face. This distance defines the location of the contact discontinuity in the statement of the CRP.

The numerical flux for the mixed cell is determined by solving in the general case two CRPs, for the first and second component, respectively. Defining the following distances:

$$
\begin{aligned}
& \delta_{1}=h \min \left(1, \frac{z}{z_{\sigma}}\right), \quad \delta_{n, 1}=h_{n} \min \left(1, \frac{z_{n}}{z_{\sigma}}\right), \\
& \delta_{2}=h \min \left(1, \frac{1-z}{1-z_{\sigma}}\right), \quad \delta_{n, 2}=h_{n} \min \left(1, \frac{1-z_{n}}{1-z_{\sigma}}\right),
\end{aligned}
$$

where $h=V / S_{\sigma}, h_{n}=V_{n} / S_{\sigma}$, the numerical flux is determined as

\footnotetext{
${ }^{1}$ A. Serejkin, privet communication, 2021
} 


$$
\mathbf{F}_{\sigma}=\mathbf{F}^{C R P}\left(\mathbf{Z}_{1}, \mathbf{Z}_{n, 1}, \delta_{1}, \delta_{n, 1}\right) z_{\sigma}+\mathbf{F}^{C R P}\left(\mathbf{Z}_{2}, \mathbf{Z}_{n, 2}, \delta_{2}, \delta_{n, 2}\right)\left(1-z_{\sigma}\right)
$$

Here we introduce the vector of primitive variables of pure components $\mathbf{Z}_{1}=\left(\rho_{1} \rho_{2} U V W p 1\right)$ and $\mathbf{Z}_{2}=\left(\rho_{1} \rho_{2} U V W p 0\right)$, and by $\mathbf{F}^{C R P}$ denote the CRP numerical flux that is introduced in the next section. Note that depending on the relation between the distance $\delta$ and the cell size $h$, there are several possible situation to occur. If $\delta_{1}=h$ and $\delta_{n, 1}=h_{n}$, the CRP is reduced to the conventional RP for the first component, and the flux across $z_{\sigma} S_{\sigma}$ is calculated with the RP solution,

$$
\mathbf{F}^{C R P}\left(\mathbf{Z}_{1}, \mathbf{Z}_{n, 1}, \delta_{1}, \delta_{n, 1}\right)=\mathbf{F}\left[\mathbf{Z}^{R}\left(\mathbf{Z}_{1}, \mathbf{Z}_{n, 1}\right)\right] .
$$

If $\delta_{1}<h$ and $\delta_{n, 1}=h_{n}$, we have the left-side CRP (the contact point is located on the left of the point of initial discontinuity. Oppositely, if $\delta_{1}=h$ and $\delta_{n, 1}<h_{n}$, it leads to the right-side CRP (the contact point on the right) which can be treated as the left-side one by reversing the cells and changing the sign of $U$. The same is true of the flux across the part of the cell face given by $\left(1-z_{\sigma}\right) S_{\sigma}$. Thus, to realize this approach, one needs to estimate the CRP flux; let it be the case of left-side CRP.

\section{CRP SOLVER}

The CRP is stated

\section{constant initial data as}
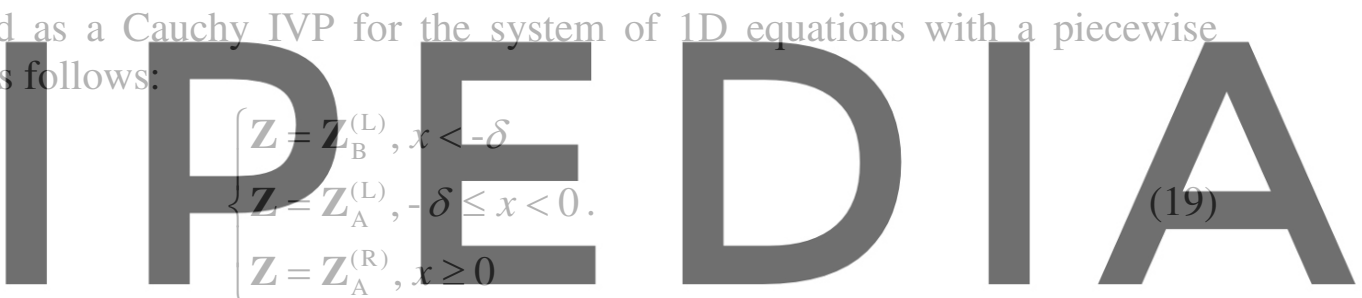

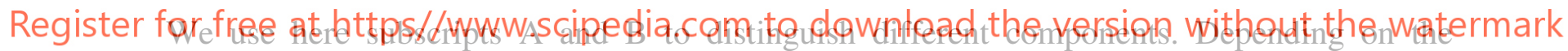
situation, A may indicate the first component $(z=1)$ and $\mathrm{B}-$ the second $(z=0)$ or vice versa.

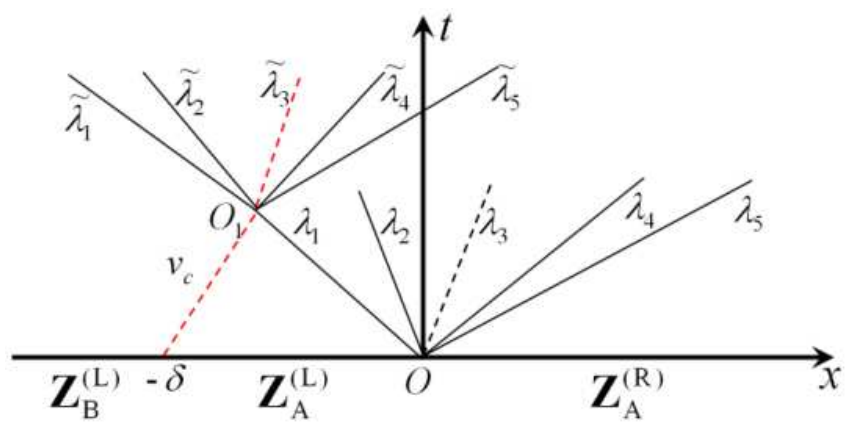

Fig. 2. Primary and secondary waves originated in the CRP solution.

The CRP solution $\mathbf{Z}^{C R P}$ represents the interaction of the waves originated due to breakup of the initial discontinuity in the material A at $x=0$ with the contact discontinuity located initially at $x=-\delta$. Decay of the initial discontinuity $(x=0)$ results in a set of primary waves 
$\lambda_{1}, \ldots, \lambda_{5}$ (see Fig. 2), where $\lambda_{3}$ is the contact discontinuity, $\lambda_{1}$ and $\lambda_{2}$ correspond to the left shock or rarefaction wave, $\lambda_{4}$ and $\lambda_{5}$ denote the characteristics of the right rarefaction wave or shock wave.

Propagating towards the material contact $v_{\mathrm{c}}$, the leftmost wave $\lambda_{1}$ interacts with the material contact at a point $O_{1}\left(t_{1}, x_{1}\right)$ (Fig. 2), with a set of secondary waves $\tilde{\lambda}_{1}, \ldots, \tilde{\lambda}_{5}$ being arisen. These waves interact with primary those with the formation tertiary set of waves and so on. The CRP solution is not self-similar and the solution along the line $x=0$ in the $x$ - $t$ plane $\mathbf{Z}_{\sigma}=\mathbf{Z}^{C R P}(x=0, t)$ is not constant. The numerical flux $\mathbf{F}_{\sigma}$ should be therefore defined by the integral $\frac{1}{\Delta t} \int_{\Delta t} \mathbf{F}\left(\mathbf{Z}_{\sigma}\right) d t$, where $\Delta t$ is the time step.

To estimate this flux we use an approximate CRP solver that takes into account only primery and secondary waves. This solution consists of the solutions to two conventional RPs. One is the primary RP stated at the point $O$ with $\mathbb{Z}_{A}^{(L)}$ and $\mathbb{Z}_{A}^{(R)}$ as initial data for $t=0$. Another is the secondary RP stated at the point of the waves interaction $O_{1}$ (Fig. 2) with $\mathbf{Z}_{B}^{(L)}$ and $\mathbf{Z}_{A}^{\text {ave }}$ as initial data for $t=t_{1}$, where $\mathbf{Z}_{A}^{\text {ave }}$ is the primitive vector of the material $\mathrm{A}$ averaged over the disturbed domain in the primery RP.

The primary and secondary RPs are solved with the HLL and HLLC approximate solvers, respectively. The HLL solver gives the averaged solution and flux in the disturbed region
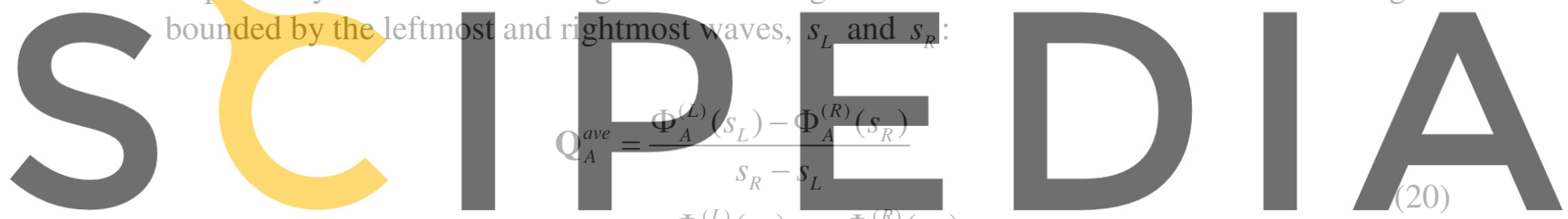

Register for free at https//www.scipedia.com to download the version without the watermark

where $\Phi(s)=\mathbb{F}-s \mathbf{Q}$, and the sub- and superscript indicate the material and the domain for which the vectors $\mathbf{F}$ and $\mathbf{Q}$ are calculated. A property of the vector $\Phi$ is that the integral $\oint_{\Gamma} \Phi(\dot{x}) d t=0$ on the solution for any closed countor in the $(x, t)$ plane. Moreover, $\Phi$ is a continuous function for discontinuous solutions $\mathbf{Q}$. The approximate CRP solver is constructed to meet these conditions.

The HLL solution represents the CRP solution for $t \leq t_{1}$ where $\Phi$ is defined as

$$
\Phi(s)=\Phi_{A}^{(L)}(s)+\left[\Phi_{A}^{a v e}(s)-\Phi_{A}^{(L)}(s)\right] H\left(s-s_{L}\right)+\left[\Phi_{A}^{(R)}(s)-\Phi_{A}^{\text {ave }}(s)\right] H\left(s-s_{R}\right)
$$

where $H(x)$ is the Heaviside function.

The secondary RP yields the CRP solution for $t>t_{1}$. It is a self-similar problem with respect to $\tilde{s}=\left(x-x_{1}\right) /\left(t-t_{1}\right)$. Its approximate solution is determined by the HLLC method 
and represented by three waves $\tilde{s}_{L}, \tilde{s}_{*}$, and $\tilde{s}_{R}$ and two disturbed zones: $\tilde{\mathbf{Q}}_{B}^{\text {ave }}$ for $\tilde{s}_{L} \leq \tilde{s} \leq \tilde{s}_{*}$ and $\tilde{\mathbf{Q}}_{A}^{\text {ave }}$ for $\tilde{s}_{*} \leq \tilde{s} \leq \tilde{s}_{R}$. The vectors of disturbed zones are given by

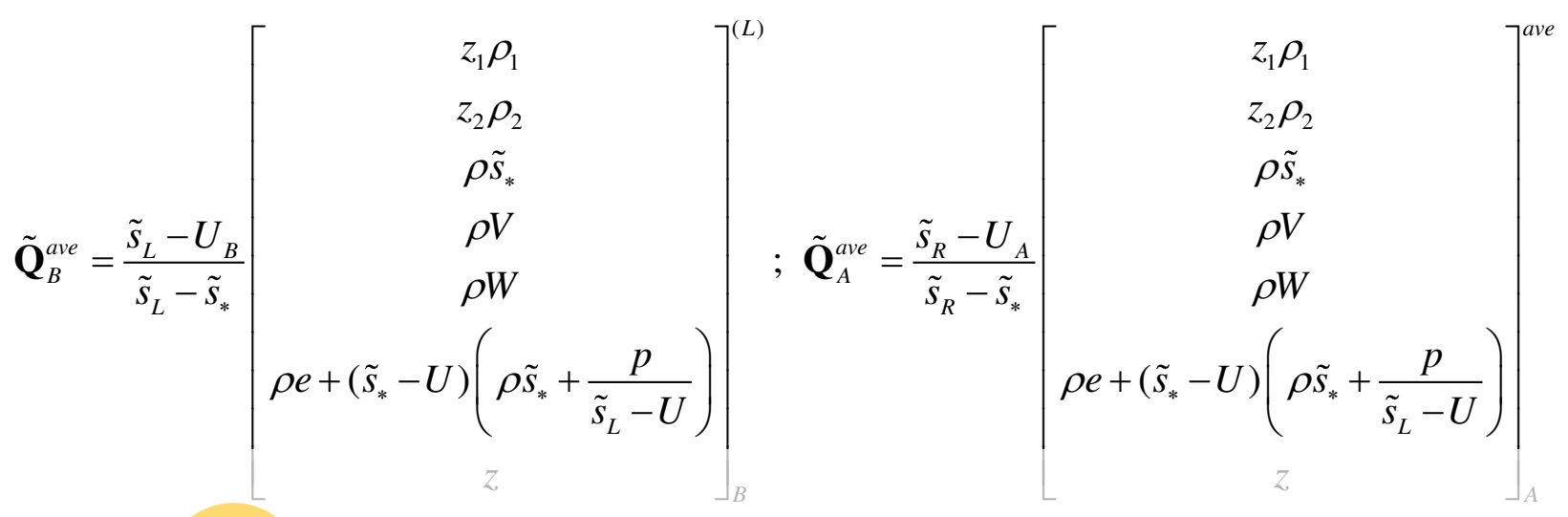

\section{The yector $\tilde{\Phi}(\tilde{s})$ that represents the HLLC solution of the secondary RP is written as}

$$
\tilde{\Phi}(\tilde{s})=\Phi_{B}^{(L)}(\tilde{s})+\left[\tilde{\Phi}_{B}^{\text {ave }}(\tilde{s})-\Phi_{B}^{(L)}(\tilde{s})\right] H\left(\tilde{s}-\tilde{S}_{L}\right)+
$$
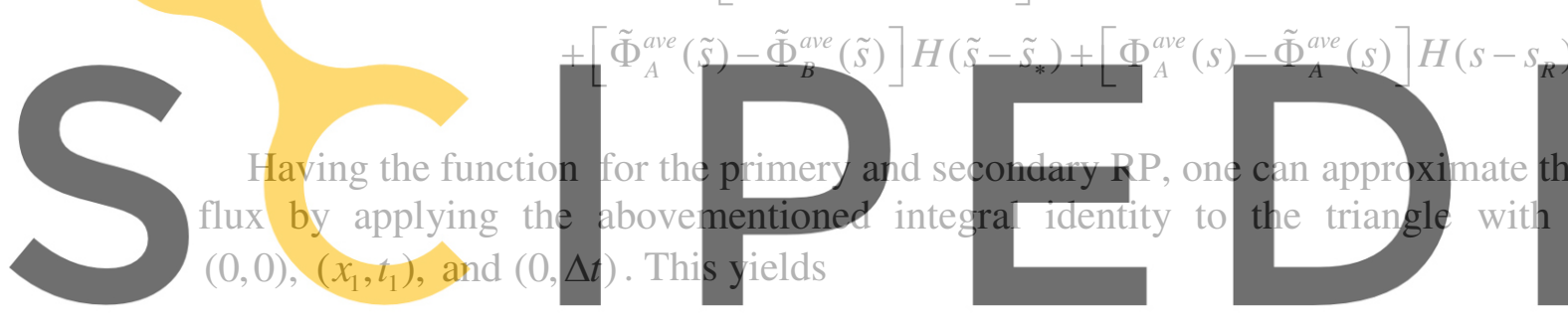

This is valid if the wave interaction time is less then the time step, $t_{1}<\Delta t$. Otherwise, the interaction happens out the time step so that there is no need to solve the CRP; the numerical flux is defined by the primery RP and can be calculated as

$$
\mathbf{F}_{\sigma}=\left\{\begin{array}{c}
\frac{\delta}{v_{c} \Delta t} \Phi_{A}^{(L)}(0)+\left(1-\frac{\delta}{v_{c} \Delta t}\right) \Phi_{B}^{(L)}(0), \text { if } \frac{\delta}{v_{c} \Delta t} \in[0,1], \\
\Phi(0), \text { otherwise. }
\end{array}\right.
$$

The CRP method performs a rather radical sharpening process of the material interface. One can prove that in the 1D case only one mixed cell is allowed in the neighborhood of the material interface [5].

The above CRP flux approximation is obtained under the assumption that only the first wave interaction, between the leftmost wave and the contact, is taken into consideration; later 
interactions are neglected. In fact, this effect of later wave interaction can be eliminated by imposing an additional restriction on the time step. Details are given in [5].

The discretization strategy for the numerical source term in the advection equation for the volume fraction works well when it is consistent with the base discretization of the numerical fluxes [6]. For consistency purpose, the same velocity as in the convective numerical flux must be utilized in the numerical source term [10]. This yields the following face-related velocity:

$$
U_{\sigma}=\frac{t_{1}}{\Delta t}\left(U_{A}^{(R)}-s_{L}\right)+\frac{\Delta t-t_{1}}{\Delta t}\left(\tilde{U}+\frac{x_{1}}{\Delta t-t_{1}}\right)
$$

when $t_{1}<\Delta t$, and for $t_{1} \geq \Delta t$,

$$
U_{\sigma}=\left\{\begin{array}{c}
U_{A(B)}^{(L)}, \text { if } \frac{\delta}{v_{c} \Delta t} \in[0,1], \\
U_{A}^{\text {ave }}, \text { otherwise. }
\end{array}\right.
$$

\section{NUMERICAL RESULTS}

A 2D code realized the above CRP method is applied to calculate the problems of this section. The first is a 1D impact problem where a layered system consisting of lead and gas impacts over a rigid wall. The computational domain is $1 \mathrm{~cm}$ in length, $0 \leq x \leq 1 \mathrm{~cm}$. Initially, lead is in the domain 0 is at a position $\quad x=0$. Lead and gas move to domain is 1 atm. We ust Lead and gas temperatur a CFL number of 0.5 .

Register for free at https//www.scipedia.com to download the version without the watermark
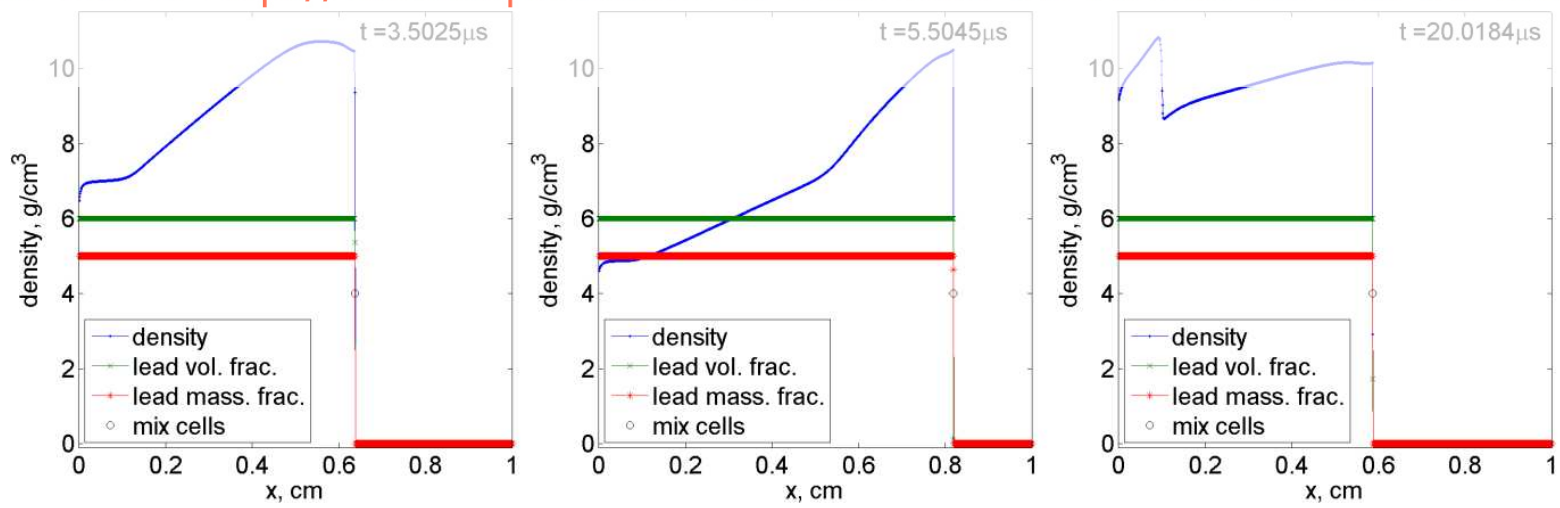

Fig. 3. Numerical results of the impact problem obtained with the CRP-based flux calculation.

In the 1D case, there should not be two neighboring mixed cells; only a "mix-pure" pattern of the sub-cell reconstruction can exist. As mentioned above, the interface in this case is resolved with only one mixed cell by the CRP method. This fact is demonstrated in Fig. 3 
where the results of calculations are shown for three different time moments. One can see that one-cell interface capturing is strictly maintained.

The efficiency in capturing highly deformed interfaces is demonstrated on the problem of underwater air bubble collapse. The problem statement is the same as in [11]. The computational domain is $0 \leq \mathrm{x} \leq 1.2 \mathrm{~cm}, 0 \leq \mathrm{y} \leq 1.2 \mathrm{~cm}$. The air bubble diameter is $0.6 \mathrm{~cm}$, and its center is at $(0.6,0.6) \mathrm{cm}$. The EOS of air and water is taken in the form of a stiffened gas EOS. Periodical boundary conditions are imposed on the bottom and top boundary of the computational domain. The CFL number is equal to 0.5. Fig. 4 shows results of calculations with the CRP method on a fine grid consisting of 2000 computational cells in each direction. This allows capturing the formation of small scale secondary jets. One can see a narrow water jet that slits the bubble and hits the water from the base.

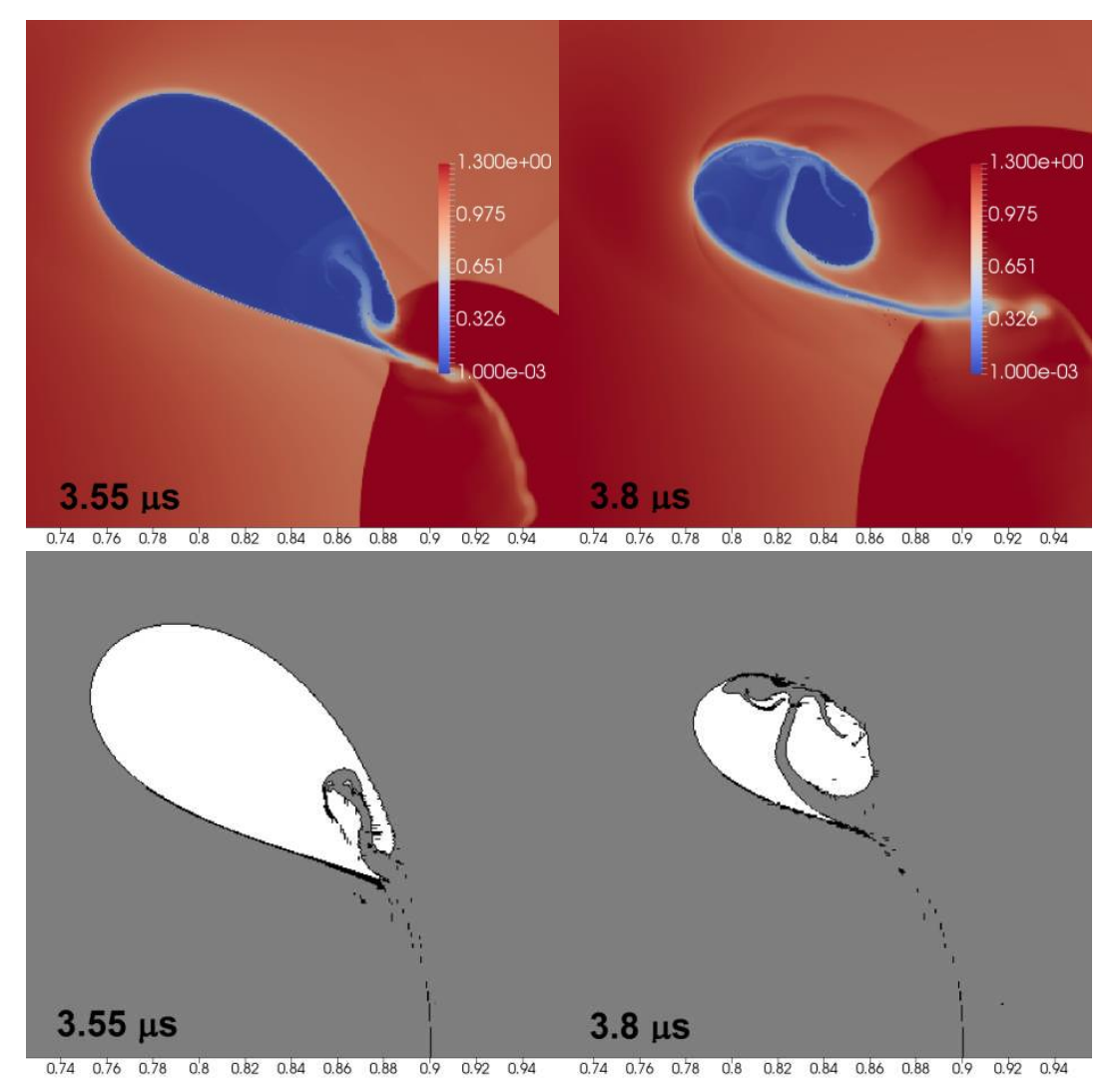

Fig. 4. The underwater air bubble collapse: the formation of secondary jets. The density distribution, $\mathrm{g} / \mathrm{cm}^{3}$ (top) and the cell type distribution (low).

\section{CONCLUSIONS}

- An Eulerian finite-volume method for two-phase fluid flows modeled by the fiveequation diffuse interface model has been considered. The method has been developed 
as an interface capturing approach and aimed to reduce numerical smearing of material interfaces.

- To achieve this goal, the sub-cell interface reconstruction procedure based on simple interfaces patterns has been introduced. The proposed sub-cell interface structure has been used for calculating the numerical flux across cell faces bordering mixed cells.

- This is performed with the Composite Riemann Problem (CRP) that involves both a point of initial discontinuity and a material contact point. A hybrid HLL-HLLC method has been incorporated to approximate the solution of the CRP with taking into account multiple wave interaction.

- The numerical flux approximation based on this CRP solution strongly reduces the interface smearing region. Numerical experiments have shown the reduction of the interface region up to one computational cell in 1D calculations and up to a few cells in $2 \mathrm{D}$ tests.

\section{REFERENCES}

[1] Harten, A. The artificial compression method for computation of shocks and contact discontinuities, I: single conservation laws. Comm. Pure Appl. Math. (1977) 30:611-638.

[2] Després, B., Lagoutière F. Contact discontinuity capturing schemes for linear advection, compressible gas dynamics. J. Sci. Comput. (2001) 16:479-524.

[3] Shyue, K.M., Xiao F. An Eulerian interface sharpening algorithm for compressible twophase flow: The algebraic THINC approach. J. Comput. Phys. (2014) 268:326-354.

[4] Menshov, I., Zakharov P. On the composite Riemann problem for multi-material fluid flows. Int. J. Numer. Meth. Fluids. (2014) 76:109-127.

[5] Zhang, Ch., Menshov I. Using the composite Riemann problem solution for capturing interfaces in compressible two-phase flows. App. Math. Comp. (2019) 363:124610.

[6] Allaire, G., Clerc, S., Kokh, S. A five-equation model for the simulation of interfaces between compressible fluids. J. Comput. Phys. (2002) 181 :577-616.

[7] Kapila, A.K., Menikoff, R., Bdzil, J.B., Son, S.F., Stewart, D.S. Two-phase modeling of deflagration-to-detonation transition in granular materials: Reduced equations. Physics of Fluids. (2001) 13 : 3002-3024.

[8] Einfeldt, B., Munz, C.D., Roe, P.L., Sjögreen, B. On Godunov-type methods near low densities. J. Comput. Phys. (1991) 92 :273-295.

[9] Batten, P., Clarke, N., Lambert, C., Causon, D.M. On the choice of wavespeeds for the HLLC Riemann solver. SIAM J. Sci. Comput. (1997) 18 :1553-1570.

[10] Coralic, V., Colonius, T. Finite-volume WENO scheme for viscous compressible multicomponent flows. J. Comput. Phys. (2014) 274 :95-121.

[11] Fedkiw, R.P., Aslam, T., Merriman, B., Osher, S. A non-oscillatory Eulerian approach to interfaces in multimaterial flows (the ghost fluid method). J. Comput. Phys. (1999) 152: $457-492$. 